\title{
Results from the DM-Ice17 Dark Matter Experiment at the South Pole
}

\author{
Jay Hyun Jo*, on behalf of the DM-Ice Collaboration \\ Department of Physics and Wright Laboratory, Yale University \\ E-mail: jayhyun.jo@yale.edu
}

DM-Ice is a phased experimental program using low-background $\mathrm{NaI}(\mathrm{Tl})$ crystals with the aim to unambiguously test the claim of dark matter detection by the DAMA experiments. DM-Ice17, consisting of $17 \mathrm{~kg}$ of $\mathrm{NaI}(\mathrm{Tl})$, has been continuously operating at a depth of $2457 \mathrm{~m}$ in the South Pole ice for over five years, demonstrating the feasibility of a low-background experiment in the Antarctic ice. Studies of low and high energy spectra, an annual modulation analysis, and a WIMP exclusion limit based on the physics run of DM-Ice17 are presented. We also discuss the plan and projected sensitivity of a new joint physics run, COSINE-100, with upgraded detectors at the Yangyang Underground Laboratory in Korea.

38th International Conference on High Energy Physics

3-10 August 2016

Chicago, USA

${ }^{*}$ Speaker. 


\section{Introduction}

Astrophysical and cosmological observations provide strong evidence that the dark matter constitutes nearly a quarter of the Universe $[1,2]$. The weakly interacting massive particle (WIMP) is a theoretically favored to explain this dark matter [3]. One method of detecting WIMP is to measure the annual modulation of a WIMP signal caused by the Earth's motion in galactic rest frame, with period of one year. Only the DAMA/NaI and DAMA/LIBRA experiments, located at Laboratori Nazionali del Gran Sasso in Italy, claim to have observed an annual modulation of dark matter accurate to $9.3 \sigma$ [4, 5], which is in conflicts with several experiments [6].

The DM-Ice experiment aims to resolve this tension by operating an experiment in the Southern Hemisphere using the same target material, thallium-doped sodium iodide ( $\mathrm{NaI}(\mathrm{Tl})$ ) scintillating crystals. While the expected dark matter modulation has the same phase everywhere on Earth, any other modulating environmental backgrounds will have $180^{\circ}$ out of phase between the Northern and Southern Hemisphere. By operating at the South Pole, we expect that DM-Ice can disentangle the dark matter phase from seasonal variations.

\section{DM-Ice17 Detector}

As the first stage of the DM-Ice experimental program, DM-Ice17 was built to demonstrate the feasibility of performing low background measurements in the Antarctic ice [7]. DM-Ice17 consists of two $8.47 \mathrm{~kg} \mathrm{NaI}(\mathrm{Tl})$ detectors, referred to as Det-1 and Det-2, which were deployed $2450 \mathrm{~m}$ into the South Pole ice in December 2010 [8]. The physics run started on June 2011 and ended on January 2015 with a total exposure of $60.8 \mathrm{~kg} \cdot \mathrm{yr}$. The detectors have continued stable operations since.

\section{Results of DM-Ice17}
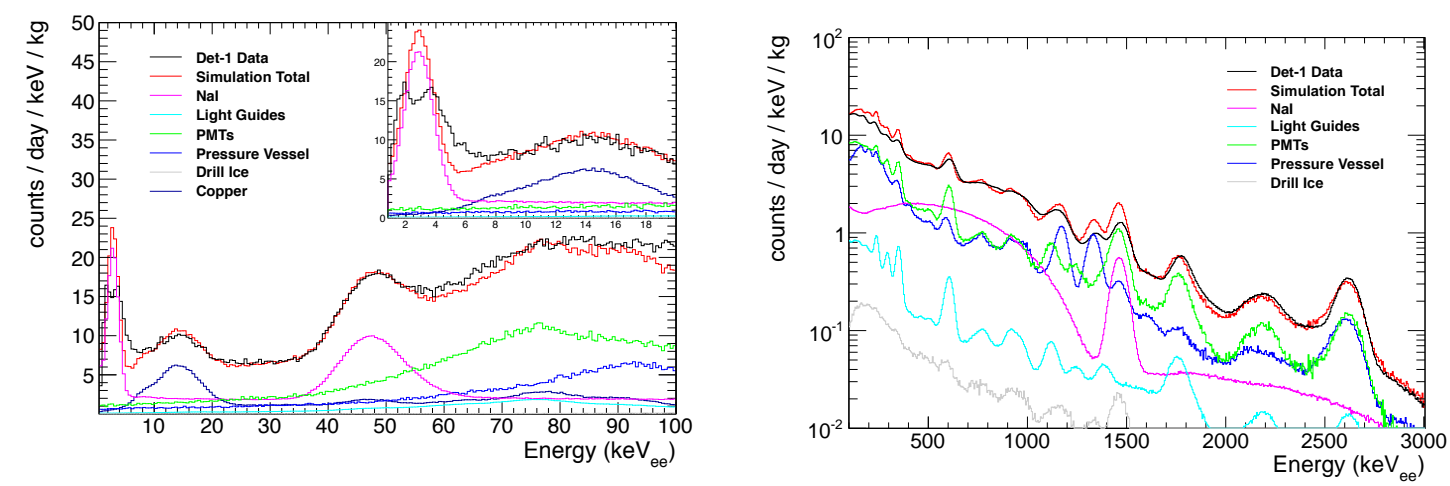

Figure 1: DM-Ice17 Det-1 background data at low (left) and high (right) energies. Detector components with main contaminations are simulated. Background peaks are used for energy calibrations.

DM-Ice17 is calibrated with intrinsic and cosmogenic backgrounds, such as ${ }^{210} \mathrm{~Pb}$ and ${ }^{125} \mathrm{I}$, respectively. Calibration measurements were performed separately within the low and high energy 
regions, due to non-linear NaI light responses. Despite inability to use external calibration sources, the ${ }^{125}$ I cosmogenic peak confirms the calibration by identifying both its energy and the decay time, with the expected half-life of 59.4 days.

Simulated background model produced with Geant 4 are consistent with the data from DMIce17, as shown in Fig. 1 [8]. At the extreme end of the low energy region, below $4 \mathrm{keV}$, simulation does not agree with the data, mainly due to the efficiency of signal retention during noise removal. Thus, an analysis threshold for Det- 1 and Det- 2 was set to $4 \mathrm{keV}$ and $6 \mathrm{keV}$, respectively.

As confirmed by simulations, the dominant sources of contamination are from the ${ }^{40} \mathrm{~K},{ }^{238} \mathrm{U}$ and ${ }^{232} \mathrm{Th}$ chains in the crystals, PMTs, and pressure vessels. The $3 \mathrm{keV}$ peak is due to Auger electrons and $\mathrm{x}$-rays from ${ }^{40} \mathrm{~K}$ decays in the crystals. A broad peak at $14 \mathrm{keV}$ can be attributed mostly to surface contamination of the ${ }^{238} \mathrm{U}$-chain in the copper encapsulation, which has been observed previously in other $\mathrm{NaI}(\mathrm{Tl})$ experiments [9]. The flat background of the crystal reaching to $30 \mathrm{keV}$ is dominated by contributions from ${ }^{210} \mathrm{~Pb}$ and ${ }^{40} \mathrm{~K}$.
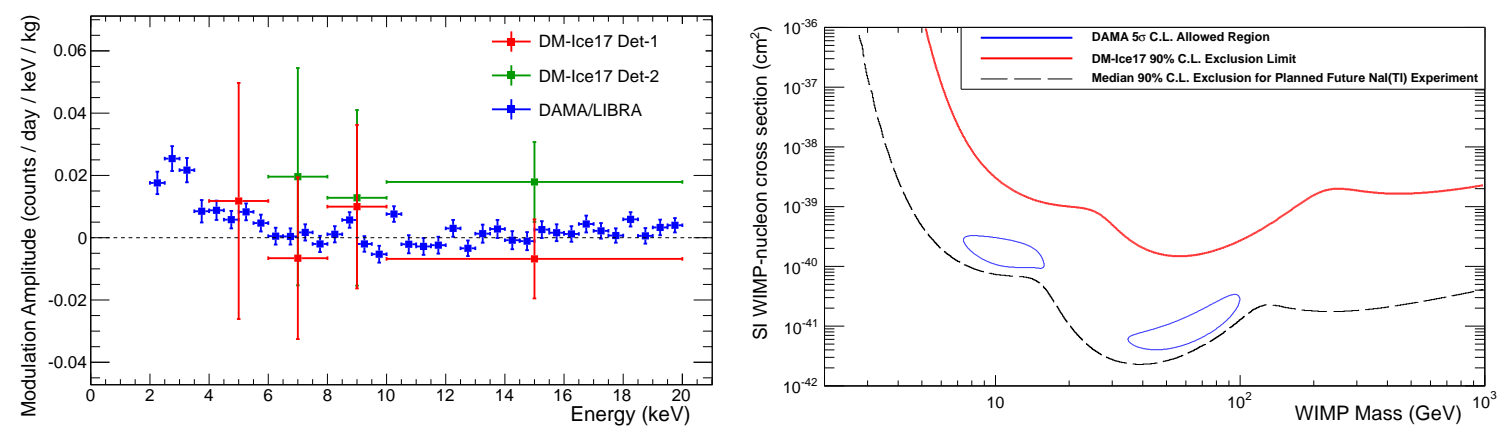

Figure 2: Left: Det-1 (red) and Det-2 (green) modulation amplitudes of each energy bin compared to the values from DAMA/LIBRA. Right: WIMP exclusion limit at $90 \%$ C.L. from the $60.8 \mathrm{~kg} \cdot \mathrm{yr}$ DM-Ice17 physics dataset (red), with DAMA preferred $5 \sigma$ C.L. contour (blue) for comparison.

Maximum likelihood fits of the background subtracted event rates for 4 different energy bins (4-6, 6-8, 8-10, and 10-20 keV) have been performed within the DM-Ice17 annual modulation analysis. When modulation amplitudes of each energy bin are compared to the values from DAMA/LIBRA with period and phase fixed to that of an expected dark matter signal (1 year and 152.5 days, respectively), as shown in Fig. 2 (left), it reveals that the data from DM-Ice17 are consistent with both the null hypothesis and DAMA/LIBRA signal under the limitations of the detector [10]. However, the result provides the strongest limit in the Southern Hemisphere by a direct detection dark matter search (See Fig. 2 (right)).

\section{Prospect: COSINE-100}

DM-Ice17 is limited by small exposure and high backgrounds, and targeted R\&D programs have been ongoing to overcome this limitation $[11,12]$. Cleaner crystals with more mass can be accessible with a new crystal vendor ${ }^{1}$ and newer PMTs with lower background and higher quantum efficiency are needed.

\footnotetext{
${ }^{1}$ http://www.alphaspectra.com
} 

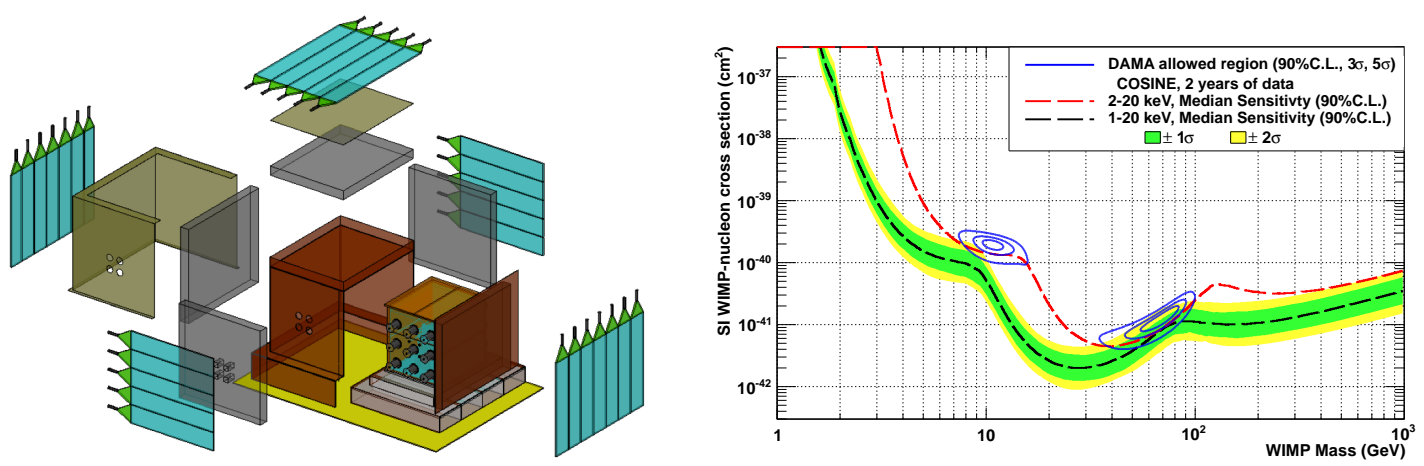

Figure 3: Left: COSINE-100 shielding structure. Right: Projected sensitivity for COSINE-100 with $1 \mathrm{keV}(2 \mathrm{keV})$ threshold in black (red) with 2 years of data.

DM-Ice and KIMS-NaI have formed a new collaboration, COSINE-100, located at Yangyang underground laboratory in South Korea. COSINE-100 consists of $8 \mathrm{NaI}(\mathrm{Tl})$ crystals with a total mass of $106 \mathrm{~kg}$ and a 2000 liter liquid scintillator veto, to help reduce low energy backgrounds by tagging ${ }^{40} \mathrm{~K}$ events. Figure 3 (left) shows the shielding structure of COSINE-100 which includes $3 \mathrm{~cm}$ of copper, $20 \mathrm{~cm}$ of lead, and $3 \mathrm{~cm}$ of 37 plastic scintillator panels for cosmic ray muon tagging. Data taking for COSINE-100 began in September 2016.

Figure 3 (right) shows projected sensitivity of COSINE-100, assuming 2-4 counts/day $/ \mathrm{keV} / \mathrm{kg}$ flat background, depending on crystal powder type. It is expected that within two years of running, COSINE-100 will achieve a sensitivity to test DAMA's result.

\section{Conclusion}

DM-Ice17, the only dark matter detector in the Southern Hemisphere, is operating successfully under Antarctic ice and established the South Pole as a site for underground low-background experiments. The DM-Ice17 physics run data, taken over 3.6 years for a total exposure of $60.8 \mathrm{~kg} \cdot \mathrm{yr}$, shows no evidence of an annual modulation in the 4-20 keV energy range. Results give the strongest exclusion limit in the Southern Hemisphere, but are consistent with both the null hypothesis and DAMA's results as DM-Ice17 is limited by exposure time and intrinsic background rates.

COSINE-100 will utilize more massive $\mathrm{NaI}(\mathrm{Tl})$ crystals with lower intrinsic backgrounds with the aid of a liquid scintillator veto. COSINE-100 has been taking data since September 2016 and two years of data will be able to test DAMA's assertion for the detection of dark matter.

\section{Acknowledgments}

The author would like to thank the conference organizers for the invitation at ICHEP2016. The DM-Ice collaboration thanks the Wisconsin IceCube Particle Astrophysics Center (WIPAC) and the IceCube collaboration for their on-going experimental support and data management. This work was supported in part by NSF Grants No. PLR-1046816, PHY-1151795, and PHY-1457995, WIPAC, the Wisconsin Alumni Research Foundation, and Yale University. 


\section{References}

[1] P. A. R. Ade et al., Astron. Astrophys. 571 (2014) A16

[2] G. Bertone, D. Hooper, and J. Silk, Phys. Rept. 405 (2005) 375

[3] G. Stegman and M. S. Turner, Nucl. Phys. B253 (1985) 375

[4] R. Bernabei et al., Eur. Phys. J. C56 (2008) 333

[5] R. Bernabei et al., Eur. Phys. J. C73 (2013) 2648

[6] D. S. Akerib et al. arXiv:1608.07648 (2016)

[7] J. Cherwinka et al., Astropart. Phys. 35 (2012) 749

[8] J. Cherwinka et al., Phys. Rev. D 90 (2014) 092005

[9] S. Cebrian et al., Astropart. Phys. 37 (2012) 60

[10] E. Barbosa de Souza et al. arXiv:1602.05939 (2016)

[11] W. C. Pettus, arXiv:1510.00378 (2015)

[12] P. Adhikari et al., Eur. Phys. J. C76 (2016) 185 